\title{
Editorial
}

\section{Machine Learning in Image Processing}

\author{
Olivier Lézoray, ${ }^{1}$ Christophe Charrier, ${ }^{1}$ Hubert Cardot, $^{2}$ and Sébastien Lefèvre ${ }^{3}$ \\ ${ }^{1}$ GREYC, UMR CNRS 6072, ENSICAEN, Université de Caen Basse-Normandie, \\ 6 Boulevard du Maréchal Juin, 14050 Caen cedex, France \\ ${ }^{2}$ Pattern Recognition and Image Analysis Team, Computer Science Laboratory (LI), Université François Rabelais de Tours, \\ 64 avenue Jean Portalis, 37200 Tours, France \\ ${ }^{3}$ Models Images Vision (MIV) Team, Image Sciences, Computer Sciences and Remote Sensing Laboratory (LSIIT), \\ Université Louis Pasteur de Strasbourg, Pôle API, Bd. Brant, BP 10413, 67412 Illkirch, France
}

Correspondence should be addressed to Olivier Lézoray, olivier.lezoray@unicaen.fr

Received 29 May 2008; Accepted 29 May 2008

Copyright (c) 2008 Olivier Lézoray et al. This is an open access article distributed under the Creative Commons Attribution License, which permits unrestricted use, distribution, and reproduction in any medium, provided the original work is properly cited.

\section{BACKGROUND AND MOTIVATION}

Images have always played an important role in human life since vision is probably human beings' most important sense. As a consequence, the field of image processing has numerous applications (medical, military, etc.). Nowadays and more than ever, images are everywhere and it is very easy for everyone to generate a huge amount of images, thanks to the advances in digital technologies. With such a profusion of images, traditional image processing techniques have to cope with more complex problems and have to face their adaptability according to human vision. With vision being complex, machine learning has emerged as a key component of intelligent computer vision programs when adaptation is needed (e.g., face recognition). With the advent of image datasets and benchmarks, machine learning and image processing have recently received a lot of attention. An innovative integration of machine learning in image processing is very likely to have a great benefit to the field, which will contribute to a better understanding of complex images. The number of image processing algorithms that incorporate some learning components is expected to increase, as adaptation is needed. However, an increase in adaptation is often linked to an increase in complexity, and one has to efficiently control any machine learning technique to properly adapt it to image processing problems. Indeed, processing huge amounts of images means being able to process huge quantities of data often of high dimensions, which is problematic for most machine learning techniques. Therefore, an interaction with the image data and with image priors is necessary to drive model selection strategies.
The primary purpose of this special issue is to increase the awareness of image processing researchers to the impact of machine learning algorithms. The special issue discusses problems and their proposed solutions currently under research by the community. In the opinion of the guest editors, the scope of this special issue covers a broad range of machine learning potentials for image processing.

\section{QUICK FACTS ABOUT THE SPECIAL ISSUE}

The guest editors suggested putting together this special issue on machine learning in image processing to the editor-inchief in November 2006. In May 2007, the guest editors and the editor-in-chief established the outline and schedule of the special issue, and the first call for papers was distributed through the Internet.

Between May and October 2007, 30 manuscripts were submitted for review and possible inclusion in the special issue.

Experts in the fields of machine learning and image processing reviewed each of the submitted manuscripts. After two rounds of rigorous reviews between November 2007 and February 2008, 14 papers were finally accepted for inclusion in the special issue.

\section{SCANNING THE SPECIAL ISSUE}

This special issue attempts to provide a comprehensive overview of the most recent trends in machine learning in image processing. The papers included in the issue focus on various topics. Accepted papers cover both theoretical 
and practical aspects of face and vehicle detection, manifold and image processing, multiresolution and multisource, and morphological processing. We organized the special issue around these topics.

\section{Face and vehicle detection}

The special issue opens with five papers on face and vehicle recognition/detection.

In their paper, "Face recognition using classificationbased linear projections," J. Goldberger and M. Butman propose a face recognition algorithm based on a linear subspace projection with neighbourhood component analysis and performance criterion to obtain the optimal linear projection.

In their paper entitled "Kernel learning of histogram of local Gabor phase patterns for face recognition," B. Zhang et al. propose a face recognition algorithm based on Daugman's method for iris recognition and the local XOR pattern operator along with kernel discriminant analysis.

In the paper entitled "Face retrieval based on robust local features and statistical-structural learning approach," I. Defee and D. Zhong propose a framework for the unification of statistical and structural information for pattern retrieval based on local feature sets.

In their paper, "DOOMRED: a new optimization technique for boosted cascade detectors on enforced training set," K. M. Lee and D. W. Park propose a new method to optimize the completely trained boosted cascade detector on an enforced training set.

In the next paper, "A cascade of boosted generative and discriminative classifiers for vehicle detection," P. Negri et al. propose an algorithm for the onboard vision vehicle detection problem using a cascade of boosted classifiers.

\section{Manifold and image processing}

The special issue continues with four papers on the processing of manifolds and images.

In their paper, "A metric multidimensional scaling-based nonlinear manifold learning approach for unsupervised data reduction," C. Heinrich et al. propose a nonlinear extension to PCA for manifold learning that makes use of compression and regression along with a Bayesian projection procedure for out-of-sample extension.

In their paper, "An adaptively accelerated Bayesian deblurring method with entropy prior," M. K. Singh et al. propose a method for image deblurring that uses a multiplicative correction term and has been calculated using an exponent on the correction factor.

In the paper entitled "Iterative estimation algorithms using conjugate function lower bound and minorizationmaximization with applications in image denoising," G. Deng and W.-Y. Ng propose a generalized algorithm for wavelet domain image denoising by solving the MAP estimation problems under a linear Gaussian observation model.

In the next paper entitled "A practical approach for simultaneous estimation of light source position, scene structure, and blind restoration using photometric observations," S. Sharma and M. Joshi propose an algorithm for photometric stereo that provides light source position and scene structure, and performs blind restoration with given observations.

\section{Multiresolution and multisource analysis}

The special issue continues with three papers making use of the multiresolution or multisource paradigms.

In their paper, "Learning how to extract rotation-invariant and scale-invariant features from texture images," J. A. Montoya-Zegarra et al. propose a texture recognition system based on steerable pyramid decomposition and optimum path forest recognition.

In the next paper, "Multiresolution image parametrization for improving texture classification,” L. Šajn and I. Kononenko present an automatic image parameterization on multiple resolutions, based on texture description with specialized association rules, and image evaluation with machine learning methods.

In their paper, "Multisource images analysis using collaborative clustering," G. Forestier et al. propose a collaborative system for image clustering by obtaining a consensus among several clusterings that exploit heterogeneous images.

\section{Morphological processing}

The special issue ends with two papers on morphological processing for segmentation and compression.

In their paper entitled "Heterogeneous stacking for classification-driven watershed segmentation," I. Levner et al. show how to design an automated segmentation system by utilizing automated feature extraction in conjunction with heterogeneous stacking for a watershed process.

Finally, in the paper entitled "Morphological transform for image compression," O. Pogrebnyak et al. present a method for image compression based on morphological associative memories.

\section{ACKNOWLEDGMENTS}

The guest editors thank all of those who have helped to make this special issue possible, especially the authors and the reviewers of the articles. They thank the editorial staff for the help and support in managing the issue, and finally gratefully acknowledge the Editor-in-Chief for giving them the opportunity to edit this special issue.

Olivier Lézoray

Christophe Charrier

Hubert Cardot

Sébastien Lefèvre 